\title{
On the Study of Multiwavelet Deconvolution Density Estimators
}

\author{
Xiaohui Zhou $\mathbb{D}^{1,2}$ \\ ${ }^{1}$ School of Mathematics, Shanghai University of Finance and Economics, Shanghai, China \\ ${ }^{2}$ Zhejiang University of Finance and Economics Dongfang College, Jiaxing, China \\ Correspondence should be addressed to Xiaohui Zhou; zhou001900@163.com
}

Received 17 September 2019; Revised 2 March 2020; Accepted 26 March 2020; Published 21 April 2020

Academic Editor: António M. Lopes

Copyright (c) 2020 Xiaohui Zhou. This is an open access article distributed under the Creative Commons Attribution License, which permits unrestricted use, distribution, and reproduction in any medium, provided the original work is properly cited.

In this paper, multiwavelet deconvolution density estimators are presented by a linear multiwavelet expansion and a nonlinear multiwavelet expansion, respectively. Moreover, the unbiased estimation is shown, and asymptotic normality is discussed for the multiwavelet deconvolution density estimators. Finally, a numerical example is given for our discussion.

\section{Introduction and Preliminary}

Assume that $(\Omega, F, P)$ is a probability space. $Y_{1}, Y_{2}, \ldots, Y_{n}$ are independent and identically distributed (i.i.d) random variables. They have the same model $Y=X+\varepsilon$, where $X$ is a real random variable and $\varepsilon$ denotes a random noise (error). Furthermore, $X$ and $\varepsilon$ are independent of each other. Let $f_{X}$ be the unknown probability density of $X$ and $f_{\varepsilon}$ be the density of $\varepsilon$. So the probability density $f_{Y}$ of $Y$ is equivalent to the convolution of $f_{X}$ and $f_{\varepsilon}$. If $f_{\varepsilon}$ degenerates to the diac functional $\delta, Y$ reduces to be noise-free. So, approximating the density $f_{X}$ by an estimator $\widehat{f}_{n}(\cdot):=\widehat{f}_{n}$ $\left(\cdot ; Y_{1}, Y_{2}, \ldots, Y_{n}\right)$ can be recognized as a deconvolution problem. A wavelet estimator $\widehat{f}_{n}$ means that $\widehat{f}_{n}$ can be expanded by a wavelet basis. Some important work has been done, such as wavelet deconvolution estimators and asymptotic normality (seen in $[1-5]$ ). Moreover, a multiwavelet estimator implies that $\widehat{f}_{n}$ can be denoted by a multiwavelet basis.

Firstly, we introduce the concept of multiplicity multiresolution analysis (MMRA) and the expansion of multiwavelet estimators. Assume that a sequence of closed subspaces $\left\{V_{j}\right\}_{j \in Z}$ in $L^{2}(R)$ satisfy the following properties:
(1) $V_{j} \subseteq V_{j+1}$
(2) $\overline{U_{j \in Z} V_{j}}=L^{2}(R), \cap_{j \in Z} V_{j}=\{0\}$
(3) $f \in V_{j} \Longleftrightarrow f(2 \cdot) \in V_{j+1}$

(4) There exists a function vector $\Phi=\left[\phi_{1}, \phi_{2}, \ldots, \phi_{r}\right]^{T}$, such that $\left\{\phi_{i}(\cdot-k), i=1, \ldots, r, k \in Z\right\}$ forms an orthogonal basis of the subspace $V_{0}$, where $V_{j}=$ clos $L^{2}(R)\left\langle\phi_{i, j, k}=2^{j / 2} \phi_{i}\left(2^{j} x-k\right): i=1,2, \ldots, r, k \in Z\right\rangle$ and $\Phi_{j, k}=\left[\phi_{1, j, k}, \phi_{2, j, k}, \ldots, \phi_{r, j, k}\right]^{T}$

For every $j \in Z$, the space $W_{j}$ can be defined as an orthogonal complement of $V_{j}$ into $V_{j+1}$, i.e., $V_{j+1}=V_{j} \oplus W_{j}$. Then, $\overline{\oplus_{j} W_{j}}=L^{2}(R)$. There exists a function vector $\Psi=$ $\left[\psi_{1}, \psi_{2}, \ldots, \psi_{r}\right]^{T} \in L^{2}(R)^{r} \quad$ such that $\left\{\psi_{i}(\cdot-k), i=\right.$ $1, \ldots, r, k \in Z\}$ forms an orthogonal basis of the subspace $W_{0}$, where $\quad W_{j}=\operatorname{clos}_{L^{2}(R)}\left\langle\psi_{i, j, k}=2^{j / 2} \psi_{i}\left(2^{j} \cdot-k\right), i=\right.$ $1,2, \ldots, r, k \in Z\rangle$. Moreover, $\Phi$ is called a multiscaling function with multiplicity $r$, and $\Psi$ is called its corresponding multiwavelet.

So, if a function $f \in L^{2}(R)$, it has the following expansion:

$$
\begin{array}{r}
f=\sum_{k \in Z} C_{j_{0} k}^{T} \Phi_{j_{0} k}+\sum_{j \geq j_{0}} \sum_{k \in Z} D_{j k}^{T} \Phi_{j k} \\
=\sum_{i=1}^{r} \sum_{k \in Z} c_{i j_{0} k} \phi_{i j_{0} k}+\sum_{i=1}^{r} \sum_{j \geq j_{0}} \sum_{k \in Z} d_{i j k} \psi_{i j k},
\end{array}
$$

where $\quad C_{j k}=\left[c_{1 j k}, c_{2 j k}, \ldots, c_{r j k}\right]^{T}, D_{j k}=\left[d_{1 j k}, d_{2 j k}, \ldots\right.$, $\left.d_{r j k}\right]^{T}, c_{i j k}=\left\langle f, \phi_{i j k}\right\rangle$, and $d_{i j k}=\left\langle f, \psi_{i j k}\right\rangle, i=1,2, \ldots, r$.

Generally, assume that $P_{j}$ and $Q_{j}$ are orthogonal projections from the space $L^{2}(R)$ to $V_{j}$ and $W_{j}$, respectively. Then, 


$$
\begin{aligned}
& P_{j} f=\sum_{k \in Z} C_{j k}^{T} \Phi_{j k}=\sum_{i=1}^{r} \sum_{k \in Z} c_{i j k} \phi_{i j k}, \\
& Q_{j} f=\sum_{k \in Z} D_{j k}^{T} \Phi_{j k}=\sum_{i=1}^{r} \sum_{k \in Z} d_{i j k} \psi_{i j k}=\left(P_{j+1}-P_{j}\right) f .
\end{aligned}
$$

Thus, $f=P_{j_{0}} f+\sum_{j=j_{0}}^{\infty} Q_{j} f$.

And we also define the notation $Q_{j_{0}, j_{1}} f=$ $\sum_{j=j_{0}}^{j_{1}} Q_{j} f=\sum_{j=j_{0}}^{j_{1}} \sum_{i=1}^{r} \sum_{k \in Z} d_{i j k} \psi_{i j k}$.

Moreover, the Fourier transform $f^{F T}$ of $f$ is defined by

$$
f^{F T}(\omega):=\int_{R} f(x) e^{-i \omega x} \mathrm{~d} x
$$

And the inverse transform of $f^{F T}$ is denoted by

$$
f(x)=\frac{1}{2 \pi} \int_{R} f^{F T}(\omega) e^{i \omega x} \mathrm{~d} \omega .
$$

So, the Fourier transform $\Phi^{F T}(\omega)$ of $\Phi(x)$ is defined as $\Phi^{F T}(\omega):=\int_{R} \Phi(x) e^{-i \omega x} \mathrm{~d} x=\left[\phi_{1}^{F T}(\omega), \phi_{2}^{F T}(\omega), \ldots, \phi_{r}^{F T}(\omega)\right]^{T}$.

In this paper, choose a multiscaling function $\Phi$ with multiplicity $r$ satisfying the following condition:

$\begin{array}{llr}\text { (C1) } \Phi=\left[\phi_{1}, \phi_{2}, \ldots, \phi_{r}\right]^{T} \in L^{2}(R)^{r} & \text { and } \\ & \left|\left(\phi_{i}^{F T}\right)^{(m)}(\omega)\right| \lesssim \quad\left(1+|\omega|^{2}\right)^{-(l / 2)} & \text { with } \\ & l>1, m=0,1,2, i=1,2, \ldots, r . & \end{array}$

Note: $A \lesssim B$ denotes two variables $A, B$ satisfying $A \leq c B$, for some constant $c>0 ; A \gtrsim B$ is equivalent to $B \lesssim A$, and $A \sim B$ means both $A \lesssim B$ and $A \gtrsim B$. Obviously, multiwavelets Sa4 (constructed by Shen et al.) [6] and CL (constructed by Chui and Lian) $[7,8]$ are examples for $\mathrm{C} 1$.

According to condition (C1), the corresponding multiwavelet $\Psi=\left[\psi_{1}, \psi_{2}, \ldots, \psi_{r}\right]^{T}$ satisfies $\left|\psi_{i}^{F T}(\omega)\right| \lesssim\left(1+|\omega|^{2}\right)^{-(l / 2)}$.

In fact, $\phi_{i}(x)=(1 / 2 \pi) \int_{R} \phi_{i}{ }^{F T}(\omega) e^{-i \omega x} \mathrm{~d} \omega$. By using integration by parts,

$$
\left|\phi_{i}(x)\right| \lesssim\left(1+|x|^{2}\right)^{-1}
$$

Then,

$$
\sup _{x \in R} \sum_{k}\left|\phi_{i}(x-k)\right| \lesssim \sup _{x \in R} \sum_{k}\left(1+|x-k|^{2}\right)^{-1} \lesssim 1 .
$$

According to multiplicity multiresolution analysis (MMRA),

$$
\sum_{k}\left|p_{i, j, k}\right|=\sum_{k}\left|\left\langle\phi_{i}, \phi_{j, 1, k}\right\rangle\right|=\sum_{k}\left|\left\langle\sqrt{2} \int_{R} \phi_{i}(x) \overline{\phi_{j}(2 x-k)} \mathrm{d} x\right\rangle\right| \lesssim 1,
$$

where $\Phi(x)=\sqrt{2} \sum_{k} P_{k} \Phi(2 x-k), P_{k}=\left(p_{i, j, k}\right)_{1 \leq, i, j \leq r}$, $\Psi(x)=\sqrt{2} \sum_{k} Q_{k} \Phi(2 x-k), Q_{k}=\left(q_{i, j, k}\right)_{1 \leq, i, j \leq r}$, $\phi_{i}(x)=\sqrt{2} \sum_{k} \sum_{j=1}^{r} p_{i, j, k} \phi_{j}(2 x-k)$, and $\psi_{i}(x)=\sqrt{2} \sum_{k}$ $\sum_{j=1}^{r} q_{i, j, k} \phi_{j}(2 x-k)$.

Moreover, $P_{i j}(\omega)=(1 / \sqrt{2}) \sum_{k} p_{i, j, k} e^{-i k \omega}$ are bounded. So, $Q_{i j}(\omega)=(1 / \sqrt{2}) \sum_{k} q_{i, j, k} e^{-i k \omega}$ are bounded, where $Q_{i j}(\omega)$ is constructed by $P_{i j}(\omega)$ (seen in [7-9]). Thus,

$$
\begin{aligned}
\left|\psi_{i}^{F T}(\omega)\right| & =\left|\sum_{j=1}^{r} Q_{i j}\left(\frac{\omega}{2}\right) \phi_{i}^{F T}\left(\frac{\omega}{2}\right)\right| \leq \sum_{j=1}^{r}\left|Q_{i j}\left(\frac{\omega}{2}\right) \phi_{i}^{F T}\left(\frac{\omega}{2}\right)\right| \\
& \lesssim\left(1+\left|\frac{\omega}{2}\right|^{2}\right)^{-(l / 2)} \lesssim\left(1+|\omega|^{2}\right)^{-(l / 2)} .
\end{aligned}
$$

In addition, the density function $f_{\varepsilon}$ of the random noise $\varepsilon$ satisfies the following conditions [2]:

(C2) $\left|f_{\varepsilon}^{F T}(\omega)\right| \gtrsim\left(1+|\omega|^{2}\right)^{-(\beta / 2)}$

(C3) $\left|\left(f_{\varepsilon}^{F T}\right)^{(m)}(\omega)\right|\left(1+|\omega|^{2}\right)^{-(\beta+m / 2)}, m=0,1,2$

Under these two conditions, the random noise $\varepsilon$ is said to be ill-posed.

\section{Multiwavelet Deconvolution Density Estimators}

In this section, we discuss the multiwavelet deconvolution density estimators. And some lemmas are deduced for the discussion of asymptotic normality in Section 3.

Similar to the discussion in $[2,3,5]$, if $l>\beta+1$, the estimators can be defined as

$$
\begin{aligned}
\widehat{c}_{i j k} & :=\frac{2^{j / 2}}{n} \sum_{p=1}^{n} K_{i j} \phi_{i}\left(2^{j} Y_{p}-k\right), \\
K_{i j} \phi_{i}(y) & :=\frac{1}{2 \pi} \int_{R} e^{i \omega y} \frac{\phi_{i}^{F T}(\omega)}{f_{\varepsilon}^{F T}\left(-2^{j} \omega\right)} \mathrm{d} \omega . \\
\widehat{d}_{i j k} & :=\frac{2^{j / 2}}{n} \sum_{p=1}^{n} K_{i j} \psi_{i}\left(2^{j} Y_{p}-k\right), \\
K_{i j} \psi_{i}(y) & :=\frac{1}{2 \pi} \int_{R} e^{i \omega y} \frac{\psi_{i}^{F T}(\omega)}{f_{\varepsilon}^{F T}\left(-2^{j} \omega\right)} \mathrm{d} \omega .
\end{aligned}
$$

According to equation (10), the linear multiwavelet estimator can be defined by

$$
\widehat{f}_{n}(x):=\sum_{i=1}^{r} \sum_{k \in Z} \widehat{c}_{i j k} \phi_{i j k} \text {. }
$$

By deducing simply, we have 


$$
\begin{aligned}
E \widehat{c}_{i j k} & =E \frac{2^{j / 2}}{n} \sum_{p=1}^{n} K_{i j} \phi_{i}\left(2^{j} Y_{p}-k\right)=\frac{2^{j / 2}}{n} \sum_{p=1}^{n} E K_{i j} \phi_{i}\left(2^{j} Y_{p}-k\right) \\
& =\frac{2^{j / 2}}{n} \sum_{p=1}^{n} \int_{R} \frac{1}{2 \pi} \int_{R} e^{i \omega\left(2^{j} Y-k\right)} \frac{\phi_{i}^{F T}(\omega)}{f_{\varepsilon}^{F T}\left(-2^{j} \omega\right)} \mathrm{d} \omega f_{Y}(Y) \mathrm{d} y \\
& =\frac{2^{j / 2}}{n 2 \pi} \sum_{p=1}^{n} \int_{R} e^{-i \omega k} \frac{\phi_{i}^{F T}(\omega)}{f_{\varepsilon}^{F T}\left(-2^{j} \omega\right)} f_{Y}^{F T}\left(-2^{j} \omega\right) \mathrm{d} \omega \\
& =\frac{2^{j / 2}}{n 2 \pi} \sum_{p=1}^{n} \int_{R} e^{-i \omega k} \phi_{i}^{F T}(\omega) f_{X}^{F T}\left(-2^{j} \omega\right) \mathrm{d} \omega \\
& =\frac{1}{n} \sum_{p=1}^{n} \int_{R} f_{X}(x) \overline{2^{j / 2} \phi_{i}\left(2^{j} x-k\right)} \mathrm{d} \omega \\
& =c_{i j k} .
\end{aligned}
$$

So $\quad E \widehat{f}_{n}(x)=E \sum_{i=1}^{r} \sum_{k \in Z} \widehat{c}_{i j k} \phi_{i j k}=\sum_{i=1}^{r} \sum_{k \in Z} c_{i j k} \phi_{i j k}=$ $P_{j} f_{X}$. And we have the following conclusion.

Theorem 1. Assume that $\widehat{c}_{i j k}$ is defined in equation (10). Then, $\widehat{c}_{i j k}$ is an unbiased estimation of $c_{i j k}=\int_{R} f_{X}$ $(x) \overline{2^{j / 2} \phi_{i}\left(2^{j} x-k\right)} d \omega$, i.e., $E \widehat{c}_{i j k}=c_{i j k}$ and $E \widehat{f}_{n}(x)=P_{j} f_{X}$.

The detailed proof of Theorem 1 is similar to the proof of Lemma 2.2 in [1].

According to the definition of $\widehat{c}_{i j k}$ in equation (10), the estimator $\widehat{f}_{n}(x)=\sum_{i=1}^{r} \sum_{k \in Z} \widehat{\widehat{c}}_{i j k} \phi_{i j k}$ can be rewritten as

$$
\widehat{f}_{n}(x)=\frac{2^{j}}{n} \sum_{i=1}^{r} \sum_{k \in Z} \sum_{p=1}^{n}\left(K_{i j} \phi_{i}\right)\left(2^{j} Y_{p}-k\right) \phi_{i}\left(2^{j} x-k\right) .
$$

To simplify the above expansion, the function

$$
K_{i}^{*}(x, y):=\sum_{k \in Z}\left(K_{i j} \phi_{i}\right)(x-k) \phi_{i}(y-k)
$$

is introduced. It is similar to the discussion in $[2,3,5]$. Then,

$$
\widehat{f}_{n}(x)=\frac{2^{j}}{n} \sum_{i=1}^{r} \sum_{p=1}^{n} K_{i}^{*}\left(2^{j} Y_{p}, 2^{j} x\right) .
$$
Then,

We introduce $\widetilde{K}_{i}^{*}(Y, x):=K_{i}^{*}(Y, x)-E K_{i}^{*}(Y, x)$.

$$
\widehat{f}_{n}(x)-E \widehat{f}_{n}(x)=\frac{2^{j}}{n} \sum_{i=1}^{r} \sum_{p=1}^{n} \widetilde{K}_{i}^{*}\left(2^{j} Y_{p}, 2^{j} x\right) .
$$

Next, the properties of the above functions are discussed in the following lemmas. Some conclusions are similar to the discussion in $[2,3,5]$.

Lemma 1. For $l>\beta+1, \beta>1$, the conditions (C1-C3) hold. Then, for every $i=1,2, \ldots, r, K_{i j} \phi_{i}$ satisfies:

$$
\left|K_{i j} \phi_{i}\right| \lesssim 2^{j \beta}\left(1+|y|^{2}\right)^{-1} .
$$

Proof. Assume that $\zeta_{i}(\omega):=\left(\phi_{i}^{F T}(\omega) / f_{\varepsilon}^{F T}\left(-2^{j} \omega\right)\right)$, for every $i=1,2, \ldots, r$. Then,

$$
K_{i j} \phi_{i}(y)=\frac{1}{2 \pi} \int_{R} e^{i \omega y} \zeta_{i}(\omega) \mathrm{d} \omega .
$$

Since

$\left|f_{\varepsilon}^{F T}(\omega)\right| \gtrsim\left(1+|\omega|^{2}\right)^{-\beta / 2} \Longrightarrow\left|f_{\varepsilon}^{F T}\left(-2^{j} \omega\right)\right| \gtrsim 2^{-j \beta}\left(1+|\omega|^{2}\right)^{-\beta / 2}$

and $\left|\left(\phi_{i}^{F T}\right)(\omega)\right| \lesssim\left(1+|\omega|^{2}\right)^{-l / 2}$,

$$
\left|\zeta_{i}(\omega)\right| \lesssim 2^{j \beta}\left(1+|\omega|^{2}\right)^{-(l-\beta / 2)} \longrightarrow 0, \quad \omega \longrightarrow \infty .
$$

We compute the derivative

$$
\begin{aligned}
\zeta_{i}^{\prime}(\omega)= & \left(\phi_{i}^{F T}\right)^{\prime}(\omega)\left[f_{\varepsilon}^{F T} 4\left(-2^{j} \omega\right)\right]^{-1}+2^{j} \phi_{i}^{F T}(\omega)\left(f_{\varepsilon}^{F T}\right)^{\prime} \\
& \cdot\left(-2^{j} \omega\right)\left[f_{\varepsilon}^{F T}\left(-2^{j} \omega\right)\right]^{-2} .
\end{aligned}
$$

According to the conditions (C1-C3) and $l>\beta+1, \beta>1$,

$$
\left|\zeta_{i}^{\prime}(\omega)\right| \lesssim\left(1+|\omega|^{2}\right)^{-l / 2}\left[\left(1+\left|2^{j} \omega\right|^{2}\right)^{\beta / 2}+2^{j}\left(1+\left|2^{j} \omega\right|^{2}\right)^{(\beta-1 / 2)}\right]
$$

$$
\longrightarrow 0, \omega \longrightarrow \infty \text {. }
$$

Similarly,

$$
\begin{aligned}
\zeta_{i}^{\prime \prime}(\omega)= & \left(\phi_{i}^{F T}\right)^{\prime \prime}(\omega)\left[f_{\varepsilon}^{F T}\left(-2^{j} \omega\right)\right]^{-1}+2^{j+1}\left(\phi_{i}^{F T}\right)^{\prime}(\omega)\left(f_{\varepsilon}^{F T}\right)^{\prime} \\
& \cdot\left(-2^{j} \omega\right)\left[f_{\varepsilon}^{F T}\left(-2^{j} \omega\right)\right]^{-2} \\
& -2^{2 j} \phi_{i}^{F T}(\omega)\left(f_{\varepsilon}^{F T}\right)^{\prime \prime}\left(-2^{j} \omega\right)\left[f_{\varepsilon}^{F T}\left(-2^{j} \omega\right)\right]^{-2} \\
& +2^{2 j+1} \phi_{i}^{F T}(\omega)\left[\left(f_{\varepsilon}^{F T}\right)^{\prime}\left(-2^{j} \omega\right)\right]^{2}\left[f_{\varepsilon}^{F T}\left(-2^{j} \omega\right)\right]^{-3} .
\end{aligned}
$$

Then,

$$
\begin{aligned}
\left|\zeta_{i}^{\prime \prime}(\omega)\right| \lesssim & \left(1+|\omega|^{2}\right)^{-l / 2}\left[\left(1+\left|2^{j} \omega\right|^{2}\right)^{\beta / 2}+2^{j+1}\left(1+\left|2^{j} \omega\right|^{2}\right)^{-(\beta+1 / 2)}\right. \\
& \cdot\left(1+\left|2^{j} \omega\right|^{2}\right)^{\beta} \\
& +2^{2 j}\left(1+\left|2^{j} \omega\right|^{2}\right)^{-(\beta+2 / 2)}\left(1+\left|2^{j} \omega\right|^{2}\right)^{\beta}+2^{2 j+1} \\
& \left.\cdot\left(1+\left|2^{j} \omega\right|^{2}\right)^{-(\beta+1)}\left(1+\left|2^{j} \omega\right|^{2}\right)^{3 \beta / 2}\right] \\
\left|\zeta_{i}^{\prime \prime}(\omega)\right| \lesssim & 2^{j \beta}\left(1+|\omega|^{2}\right)^{-(l / 2)}\left[\left(2^{-2 j}+|\omega|^{2}\right)^{\beta / 2}+\left(2^{-2 j}+|\omega|^{2}\right)^{\beta-1 / 2}\right. \\
& \left.+\left(2^{-2 j}+|\omega|^{2}\right)^{\beta-2 / 2}\right] .
\end{aligned}
$$

So, the derivative functions $\zeta_{i}^{\prime}(\omega)$ and $\zeta_{i}^{\prime \prime}(\omega)$ are bounded. By integration by parts, 
$K_{i j} \phi_{i}(y)=\frac{1}{2 \pi} \int_{R} e^{i \omega y} \zeta_{i}(\omega) \mathrm{d} \omega=\frac{1}{2 \pi(i y)^{2}} \int_{R} e^{i \omega y}\left(\zeta_{i}\right)^{\prime \prime}(\omega) \mathrm{d} \omega$.

If $|y|<1$, it is obtained that

$$
\begin{aligned}
\left|K_{i j} \phi_{i}(y)\right| & \lesssim \int_{R}\left|\zeta_{i}(\omega)\right| \mathrm{d} \omega \leqslant 2^{j \beta} \int_{R}\left(1+|\omega|^{2}\right)^{-l-\beta / 2} \mathrm{~d} \omega \lesssim 2^{j \beta} \\
& \lesssim 2^{j \beta}\left(1+|y|^{2}\right)^{-1} .
\end{aligned}
$$

If $|y| \geq 1$, the conclusion holds that

$$
\left|K_{i j} \phi_{i}(y)\right| \lesssim|y|^{-2} \int_{R} \zeta_{i}^{\prime \prime}|(\omega)| \mathrm{d} \omega \lesssim\left(1+|y|^{2}\right)^{-1} \int_{R}\left|\zeta_{i}^{\prime \prime}(\omega)\right| \mathrm{d} \omega .
$$

For every $j \in N, \quad \mathfrak{R}_{j}:=\left\{\left.\omega\left|2^{-2 j}+\right| \omega\right|^{2} \geq 1\right\} \quad$ and $\Re_{j}^{c}=\left\{\left.\omega\left|2^{-2 j}+\right| \omega\right|^{2}<1\right\}$.

If $\omega \in \mathfrak{R}_{j}$,

$$
\left|\zeta_{i}^{\prime \prime}(\omega)\right| \lesssim 2^{j \beta}\left(1+|\omega|^{2}\right)^{-(l / 2)}\left(2^{-2 j}+|\omega|^{2}\right)^{\beta / 2} \lesssim 2^{j \beta}\left(1+|\omega|^{2}\right)^{-(l-\beta / 2)} .
$$

Then,

$$
\begin{aligned}
\int_{\mathfrak{R}_{j}}\left|\zeta_{i}^{\prime \prime}(\omega)\right| v \mathrm{~d} \omega & \lesssim 2^{j \beta} \int_{\mathfrak{R}_{j}}\left(1+|\omega|^{2}\right)^{-(l-\beta / 2)} \mathrm{d} \omega \\
& \lesssim 2^{j \beta} \int_{R}\left(1+|\omega|^{2}\right)^{-(l-\beta / 2)} \mathrm{d} \omega \lesssim 2^{j \beta} .
\end{aligned}
$$

If $\omega \in \mathfrak{R}_{j}^{c}$,

$\left|\zeta_{i}^{\prime \prime}(\omega)\right| \lesssim 2^{j \beta}\left(1+|\omega|^{2}\right)^{-(l / 2)}\left(2^{-2 j}+|\omega|^{2}\right)^{(\beta-2 / 2)} \leqslant 2^{j \beta}\left(2^{-2 j}+|\omega|^{2}\right)^{(\beta-2 / 2)}$.

Thus, for $\beta \geq 2$,

$\int_{\Re_{j}^{c}}\left|\zeta_{i}^{\prime \prime}(\omega)\right| \mathrm{d} \omega \leq \int_{|\omega|<1}\left|\zeta_{i}^{\prime \prime}(\omega)\right| \mathrm{d} \omega \leq 2^{j \beta} \int_{|\omega|<1}\left(1+|\omega|^{2}\right)^{(\beta-2 / 2)} \mathrm{d} \omega \lesssim 2^{j \beta}$.

And if $1<\beta<2$,

$\int_{\Re_{j}^{c}}\left|\zeta_{i}^{\prime \prime}(\omega)\right| \mathrm{d} \omega \leq \int_{|\omega|<1}\left|\zeta_{i}^{\prime \prime}(\omega)\right| \mathrm{d} \omega \lesssim 2^{j \beta} \int_{0}^{1}|\omega|^{\beta-2} \mathrm{~d} \omega \lesssim 2^{j \beta}$.

So, for every $\beta>1$,

$$
\int_{R_{j}^{c}}\left|\zeta_{i}^{\prime \prime}(\omega)\right| \mathrm{d} \omega \lesssim 2^{j \beta}
$$

Hence, for every $|y| \geq 1$,

$$
\left|K_{i j} \phi_{i}(y)\right| \lesssim 2^{j \beta}\left(1+|y|^{2}\right)^{-1} \text {. }
$$

The conclusion is similar to Lemma 2.1 in [2]. According to the above conclusion, we have the following lemma.

Lemma 2. For $l>\beta+1, \beta>1$, under the conditions (C1-C3), define the function $F(|x|):=\left(1+|x|^{-1}\right)$. Then, for every $i=1,2, \ldots, r, K_{i j} \phi_{i}$ and $K_{i}^{*}$ satisfy the following:
(1) $\left|K_{i}^{*}(x, y)\right| \lesssim 2^{j \beta} F(|x-y|)$

(2) $\sum_{k \in Z}\left(K_{i j} \phi_{i}\right)(x-k) K_{i^{\prime} j} \phi_{i^{\prime}}(y-k) \lesssim 2^{j \beta} F(|x-y|)$

(3) $\sum_{i=1}^{r} E K_{i}^{*}\left(2^{j} x, 2^{j} y\right)=2^{-j} P_{j} f_{X}(x)\left(f_{X} \in L^{2}(R)\right)$, where $P_{j} f_{X}$ is defined in equation (2)

Proof. According to the conclusion of Lemma 1,

$$
\left|K_{i j} \phi_{i}(x-k)\right| \lesssim 2^{j \beta}\left(1+|x-k|^{2}\right)^{-1},
$$

for every $i=1,2, \ldots, r$. Then,

$$
\sup _{x \in R} \sum_{k \in Z}\left|K_{i j} \phi_{i}(x-k)\right| \lesssim 2^{j \beta},
$$

where $Z=\{k \in Z,|x-k| \geq(|x-y| / 2)\} \quad \cup\{k \in Z,|y-k| \geq$ $(|x-y| / 2)\}$ for fixed $x, y \in R$.

According to the definition of $K_{i}^{*}(x, y)$,

$$
\begin{aligned}
\left|K_{i}^{*}(x, y)\right| \leq & \sum_{|x-k| \geq(|x-y| / 2)}\left|\left(K_{i j} \phi_{i}\right)(x-k)\right|\left|\phi_{i}(y-k)\right| \\
& +\sum_{|y-k| \geq(|x-y| / 2)}\left|\left(K_{i j} \phi_{i}\right)(x-k)\right|\left|\phi_{i}(y-k)\right| .
\end{aligned}
$$

On the other hand, $\phi_{i}(x)=(1 / 2 \pi) \int_{R} \phi_{i}^{F T}(\omega) e^{i \omega x} \mathrm{~d} \omega$.

According to the condition $\mathrm{C} 1$ and integration by parts, we have

$$
\left|\phi_{i}(x)\right| \lesssim\left(1+|x|^{2}\right)^{-1}=F(|x|),
$$

that is,

$$
\left|\phi_{i}(y-k)\right| \lesssim F(|y-k|) \lesssim F(|x-y|) .
$$

So,

$$
\sum_{|y-k| \geq(|x-y| / 2)}\left(K_{i j} \phi_{i}\right)(x-k)|| \phi_{i}(y-k) \mid \leqslant 2^{j \beta} F(|x-y|) .
$$

Since

$$
\begin{aligned}
\left|\left(K_{i j} \phi_{i}\right)(x-k)\right| & \lesssim 2^{j \beta}\left(1+|x-k|^{2}\right)^{-1}=2^{j \beta} F(|x-k|) \\
& \leq 2^{j \beta} F\left(\frac{|x-y|}{2}\right), \\
& \cdot \sum_{|x-k| \geq(|x-y| / 2)}\left|\left(K_{i j} \phi_{i}\right)(x-k)\right|\left|\phi_{i}(y-k)\right| \\
& \lesssim \sum_{k \in Z} 2^{j \beta} F\left(\frac{|x-y|}{2}\right)\left|\phi_{i}(y-k)\right| .
\end{aligned}
$$

On the other hand, $\left|\phi_{i}(x)\right| \lesssim F(|x|)$ implies $\sum_{k \in Z}\left|\phi_{i}(y-k)\right| \lesssim 1$. So the conclusion (2) holds.

For the conclusion (10), according to Lemma 1 and the above discussion in conclusion (2), 


$$
\begin{aligned}
& \left|K_{i^{\prime} j} \phi_{i^{\prime}}(y-k)\right| \leqslant 2^{j \beta}\left(1+|y-k|^{2}\right)^{-1}=2^{j \beta} F(|y-k|), \\
& \sum_{k \in Z}\left(\left(K_{i j} \phi_{i}\right)(x-k)|| K_{i^{\prime} j} \phi_{i^{\prime}}(y-k) \mid\right. \\
& \leqslant \sum_{k \in Z}\left|\left(K_{i j} \phi_{i}\right)(x-k)\right| 2^{j \beta} F(|y-k|) \leqslant 2^{2 j \beta} F(|x-y|) .
\end{aligned}
$$

Next, to prove the conclusion (11),

$$
\begin{aligned}
E K_{i}^{*}\left(2^{j} Y, 2^{j} x\right) & =\int_{R} K_{i}^{*}\left(2^{j} Y, 2^{j} x\right) f_{Y}(y) \mathrm{d} y \\
& =\int_{R} \sum_{k \in Z}\left(K_{i j} \phi_{i}\right)\left(2^{j} y-k\right) \phi_{i}\left(2^{j} x-k\right) f_{Y}(y) \mathrm{d} y .
\end{aligned}
$$

Note that $\left|\phi_{i}(x)\right| \lesssim F(|x|) \lesssim 1$, then

$$
\int_{R} \sum_{k \in Z}\left|\left(K_{i j} \phi_{i}\right)\left(2^{j} y-k\right) \phi_{i}\left(2^{j} x-k\right)\right| f_{Y}(y) \mathrm{d} y \lesssim 2^{j \beta} \int_{R} f_{Y}(y) \mathrm{d} y=2^{j \beta} .
$$

So

$$
\begin{aligned}
E K_{i}^{*}\left(2^{j} Y, 2^{j} x\right)= & \sum_{k \in Z} \int_{R}\left(K_{i j} \phi_{i}\right)\left(2^{j} y-k\right) f_{Y}(y) \mathrm{d} y \\
& \cdot \phi_{i}\left(2^{j} x-k\right) .
\end{aligned}
$$

By the definition of $K_{i j} \phi_{i}$ in equation (10) and Fubini theorem,

$$
\begin{aligned}
\int_{R}\left(K_{i j} \phi_{i}\right)\left(2^{j} y-k\right) f_{Y}(y) \mathrm{d} y & =\int_{R} \frac{1}{2 \pi} \int_{R} e^{i \omega\left(2^{j} y-k\right)} \frac{\phi_{i}^{F T}(\omega)}{f_{\varepsilon}^{F T}\left(-2^{j} \omega\right)} \mathrm{d} \omega f_{Y}(y) \mathrm{d} y \\
& =\frac{1}{2 \pi} \int_{R} e^{-i \omega k} \int_{R} e^{-i \omega\left(-2^{j} y\right)} f_{Y}(y) \mathrm{d} y \frac{\phi_{i}^{F T}(\omega)}{f_{\varepsilon}^{F T}\left(-2^{j} \omega\right)} \mathrm{d} \omega \\
& =\frac{1}{2 \pi} \int_{R} e^{-i \omega k} f_{Y}^{F T}\left(-2^{j} \omega\right) \frac{\phi_{i}^{F T}(\omega)}{f_{\varepsilon}^{F T}\left(-2^{j} \omega\right)} \mathrm{d} \omega .
\end{aligned}
$$

According to the convolution $f_{Y}^{F T}=f_{X}^{F T} \cdot f_{\varepsilon}^{F T}$,

$$
\begin{aligned}
\int_{R}\left(K_{i j} \phi_{i}\right)\left(2^{j} y-k\right) f_{Y}(y) \mathrm{d} y & =\frac{1}{2 \pi} \int_{R} f_{X}^{F T}\left(-2^{j} \omega\right) \phi_{i}^{F T}(\omega) e^{-i \omega k} \mathrm{~d} \omega \\
& =\frac{2^{-j}}{2 \pi} \int_{R} f_{X}^{F T}(\omega) \phi_{i}^{F T}\left(-2^{-j} \omega\right) e^{i 2^{-j} k} \mathrm{~d} \omega \\
& =\int_{R} f_{X}(x) \phi_{i}\left(2^{j} x-k\right) \mathrm{d} \omega .
\end{aligned}
$$

The final equality is due to Plancheral formula:

$$
\begin{aligned}
\sum_{i=1}^{r} 2^{j} E K_{i}^{*}\left(2^{j} Y, 2^{j} x\right)= & \sum_{k \in Z} \int_{R} f_{X}(x) 2^{j / 2} \phi_{i}\left(2^{j} x-k\right) \mathrm{d} \omega \\
& \cdot 2^{j / 2} \phi_{i}\left(2^{j} x-k\right) \\
= & \sum_{i=1}^{r} \sum_{k \in Z}<f_{X}, \phi_{i j k}>\phi_{i j k}(x) \\
& =P_{j} f_{X}(x) .
\end{aligned}
$$

The conclusion (11) holds.

Lemma 3 (see 2, Theorem 3.1). Assume that $H_{n}(\cdot, \cdot),(n=$ $1,2, \ldots)$ are symmetric functions, $X_{1}, X_{2}, \ldots, X_{n}$ are i.i.d random variables and $G_{n}(x, y):=E\left[H_{n}\left(x, X_{1}\right) H_{n}\left(y, X_{1}\right)\right]$.
If $E\left[H_{n}\left(X_{1}, X_{2}\right) \mid X_{1}\right]=0, E H_{n}^{2}\left(X_{1}, X_{2}\right)<\infty(n=1,2, \ldots)$, and

$$
\lim _{n \rightarrow \infty} \frac{E G_{n}^{2}\left(X_{1}, X_{2}\right)+n^{-1} E H_{n}^{4}\left(X_{1}, X_{4}\right)}{\left[E H_{n}^{2}\left(X_{1}, X_{4}\right)\right]^{2}}=0 .
$$

Then, $s_{n}^{-1} U_{n} \stackrel{d}{\longrightarrow} N(0,1)$, where $U_{n}:=\sum_{1 \leq i<j \leq n} H_{n}$ $\left(X_{i}, X_{j}\right)$ and $s_{n}^{2}:=E U_{n}^{2}=(1 / 2) n(n-1) E H_{n}^{2}\left(X_{1}, X_{2}\right)$.

\section{Asymptotic Normality}

In this section, asymptotic normality is discussed for the linear multiwavelet deconvolution estimator $\widehat{f}_{n}(x)$ and the nonlinear estimator $\widehat{f}_{n}^{\text {non }}(x)$.

Theorem 2. Under the condition (C1-C3), with $\beta>1$ and $l>\beta+1$, if $f_{X} \in L^{p}(R),(p>2)$, then the linear estimator $\widehat{f}_{n}$ satisfies 


$$
n 2^{-j(2 \beta+(1 / 2))}\left[\left\|\hat{f}_{n}-f_{X}\right\|_{2}^{2}-E\left\|\hat{f}_{n}-f_{X}\right\|_{2}^{2}\right] \stackrel{d}{\longrightarrow} N\left(0, \sum_{1 \leq i_{1}<i_{2} \leq r} s_{i_{1}, i_{2}, n}^{2}\right),
$$

with $j \longrightarrow \infty$ and $n^{-1} 2^{j} \longrightarrow 0$, where $s_{i_{1}, i_{2}, n}^{2}:=(1 / 2) n(n-$ 1) $E H_{i_{1}, i_{2}, n}^{2}\left(Y_{1}, Y_{2}\right)$ and $H_{i_{1}, i_{2}, n}^{2}\left(Y_{p_{1}}, Y_{p_{2}}\right)$ are defined by equations (55) and (62).

Proof. Since $E \widehat{f}_{n}(x)=P_{j} f, \widehat{f}_{n}(x)-E \widehat{f}_{n}(x) \in V_{j}$ and

$$
E \widehat{f}_{n}-f_{X}=-\sum_{j^{\prime}=j}^{\infty} Q_{j^{\prime}} f_{X}
$$

So $\widehat{f}_{n}(x)-E \hat{f}_{n}(x)$ and $E \hat{f}_{n}-f_{X}$ are orthogonal in $L^{2}(R)$. We have

$$
\begin{aligned}
\left\|\widehat{f}_{n}-f_{X}\right\|_{2}^{2}= & \left\|\widehat{f}_{n}-E \widehat{f}_{n}+E \widehat{f}_{n}-f_{X}\right\|_{2}^{2}=\left\|\widehat{f}_{n}-E \widehat{f}_{n}\right\|_{2}^{2} \\
& +\left\|E \widehat{f}_{n}-f_{X}\right\|_{2}^{2} .
\end{aligned}
$$

Assume that

$$
\begin{aligned}
& J_{n}:=\left\|\hat{f}_{n}-f_{X}\right\|_{2}^{2}-E\left\|\hat{f}_{n}-f_{X}\right\|_{2}^{2}=\left\|\hat{f}_{n}-E \hat{f}_{n}\right\|_{2}^{2}-E\left\|\hat{f}_{n}-E \hat{f}_{n}\right\|_{2}^{2} \text {, } \\
& \left|\widehat{f}_{n}(x)-E \widehat{f}_{n}(x)\right|^{2}=\frac{2^{2 j}}{n^{2}}\left(\sum_{i=1}^{r} \sum_{p=1}^{n} \widetilde{K}_{i}^{*}\left(2^{j} Y_{p}, 2^{j} x\right)\right)^{2} \\
& =\frac{2^{2 j}}{n^{2}} \sum_{i=1}^{r} \sum_{p=1}^{n}\left(\widetilde{K}_{i}^{*}\left(2^{j} Y_{p}, 2^{j} x\right)\right)^{2} \\
& +\frac{2^{2 j}}{n^{2}} \sum_{1 \leq i_{1}<i_{2} \leq r} \sum_{1 \leq p_{1}<p_{2} \leq n}\left[\widetilde{K}_{i_{1}}^{*}\left(2^{j} Y_{p_{1}}, 2^{j} x\right) \widetilde{K}_{i_{2}}^{*}\left(2^{j} Y_{p_{2}}, 2^{j} x\right)\right. \\
& \left.+\widetilde{K}_{i_{1}}^{*}\left(2^{j} Y_{p_{2}}, 2^{j} x\right) \widetilde{K}_{i_{2}}^{*}\left(2^{j} Y_{p_{1}}, 2^{j} x\right)\right] \\
& +\frac{2^{2 j+1}}{n^{2}} \sum_{1 \leq i \leq r} \sum_{1 \leq p_{1}<p_{2} \leq n} \widetilde{K}_{i}^{*}\left(2^{j} Y_{p_{1}}, 2^{j} x\right) \widetilde{K}_{i}^{*}\left(2^{j} Y_{p_{2}}, 2^{j} x\right) \\
& +\frac{2^{2 j+1}}{n^{2}} \sum_{1 \leq i_{1}<i_{2} \leq r} \sum_{1 \leq p \leq n} \widetilde{K}_{i_{1}}^{*}\left(2^{j} Y_{p}, 2^{j} x\right) \widetilde{K}_{i_{2}}^{*}\left(2^{j} Y_{p}, 2^{j} x\right) . \\
& H_{i_{1}, i_{2}, n}^{*}\left(Y_{p_{1}}, Y_{p_{2}}\right):=\int_{R} \widetilde{K}_{i_{1}}^{*}\left(2^{j} Y_{p_{1}}, 2^{j} x\right) \widetilde{K}_{i_{2}}^{*}\left(2^{j} Y_{p_{2}}, 2^{j} x\right) \\
& +\widetilde{K}_{i_{1}}^{*}\left(2^{j} Y_{p_{2}}, 2^{j} x\right) \widetilde{K}_{i_{2}}^{*}\left(2^{j} Y_{p_{1}}, 2^{j} x\right) \mathrm{d} x . \\
& J_{n}=\frac{2^{2 j}}{n^{2}} \sum_{1 \leq i_{1}<i_{2} \leq r} \sum_{1 \leq p_{1}<p_{2} \leq n} H_{i_{1}, i_{2}, n}^{*}\left(Y_{p_{1}}, Y_{p_{2}}\right) \\
& +\frac{2^{2 j-1}}{n^{2}} \sum_{i=1}^{r} \sum_{p=1}^{n}\left[H_{i, i, n}^{*}\left(Y_{p}, Y_{p}\right)-E H_{i, i, n}^{*}\left(Y_{p}, Y_{p}\right)\right] \\
& +\frac{2^{2 j}}{n^{2}} \sum_{1 \leq i \leq r} \sum_{1 \leq p_{1}<p_{2} \leq n} H_{i, i, n}^{*}\left(Y_{p_{1}}, Y_{p_{2}}\right)
\end{aligned}
$$


According to Lemma 2 and $\widetilde{K}_{i}^{*}(Y, x):=$ Moreover,

$K_{i}^{*}(Y, x)-E K_{i}^{*}(Y, x)$, we have

$$
\begin{aligned}
\left|\widetilde{K}_{i_{1}}^{*}\left(2^{j} Y_{p_{1}}, 2^{j} x\right) \widetilde{K}_{i_{2}}^{*}\left(2^{j} Y_{p_{2}}, 2^{j} x\right)\right| \lesssim & 2^{2 j \beta} F\left(2^{j}\left|Y_{p_{1}}-x\right|\right) F \\
& \cdot\left(2^{j}\left|Y_{p_{2}}-x\right|\right), \\
\operatorname{var}\left(H_{i, i, n}^{*}\left(Y_{p}, Y_{p}\right)\right) \leq & E\left(H_{i, i, n}^{*}\left(Y_{p}, Y_{p}\right)\right)^{2} \lesssim 2^{j(4 \beta-2)}, \\
\operatorname{var}\left(H_{i_{1}, i_{2}, n}^{*}\left(Y_{p}, Y_{p}\right)\right) \leq & E\left(H_{i_{1}, i_{2}, n}^{*}\left(Y_{p}, Y_{p}\right)\right)^{2} \lesssim 2^{j(4 \beta-2)} .
\end{aligned}
$$

$$
\begin{aligned}
& \operatorname{var}\left(\sum_{i=1}^{r} H_{i, i, n}^{*}\left(Y_{p}, Y_{p}\right)\right) \leq E\left(\sum_{i=1}^{r} H_{i, i, n}^{*}\left(Y_{p}, Y_{p}\right)\right)^{2} \leq 2^{j(4 \beta-2)}, \\
& \operatorname{var}\left(\sum_{1 \leq i_{1}<i_{2} \leq r} H_{i_{1}, i_{2}, n}^{*}\left(Y_{p}, Y_{p}\right)\right) \leq E\left(\sum_{1 \leq i_{1}<i_{2} \leq r} H_{i_{1}, i_{2}, n}^{*}\left(Y_{p}, Y_{p}\right)\right)^{2} \leq 2^{j(4 \beta-2)} .
\end{aligned}
$$

By Markov's inequality, $\forall \varepsilon>0$,

$$
\begin{aligned}
& P\left\{\left|J_{n}^{(2)}\right| \geq n^{-1} 2^{j(2 \beta+(1 / 2))} \varepsilon\right\} \\
& =P\left\{\left|\sum_{i=1}^{r} \sum_{p=1}^{n}\left[H_{i, i, n}^{*}\left(Y_{p}, Y_{p}\right)-E H_{i, i, n}^{*}\left(Y_{p}, Y_{p}\right)\right]\right| \geq 2 n 2^{j(2 \beta-(3 / 2))} \varepsilon\right\} \\
& \leq P\left\{\left|\sum_{i=1}^{r} \sum_{p=1}^{n}\left[H_{i, i, n}^{*}\left(Y_{p}, Y_{p}\right)-E H_{i, i, n}^{*}\left(Y_{p}, Y_{p}\right)\right]\right| \geq n 2^{j(2 \beta-(3 / 2))} \varepsilon\right\} \\
& \leq \frac{\operatorname{var}\left(\sum_{i=1}^{r} \sum_{p=1}^{n} H_{i, i, n}^{*}\left(Y_{p}, Y_{p}\right)\right)}{n^{2} 2^{j(4 \beta-3)} \mathcal{E}^{2}}, \\
& P\left\{\left|J_{n}^{(4)}\right| \geq n^{-1} 2^{j(2 \beta+(1 / 2))} \varepsilon\right\} \\
& =P\left\{\left|\sum_{1 \leq i_{1}<i_{2} \leq r} \sum_{p=1}^{n}\left[H_{i_{1}, i_{2}, n}^{*}\left(Y_{p}, Y_{p}\right)-E H_{i_{1}, i_{2}, n}^{*}\left(Y_{p}, Y_{p}\right)\right]\right| \geq n 2^{j(2 \beta-(3 / 2))} \varepsilon\right\} \\
& \leq \frac{\operatorname{var}\left(\sum_{1 i_{1}<i_{2} \leq r} \sum_{p=1}^{n} H_{i_{1}, i_{2}, n}^{*}\left(Y_{p}, Y_{p}\right)\right)}{n^{2} 2^{j(4 \beta-3)} \varepsilon^{2}} .
\end{aligned}
$$

According to the independence of $\left\{Y_{p}\right\}_{p=1}^{n}$,

$$
\begin{gathered}
P\left\{\left|J_{n}^{(2)}\right| \geq n^{-1} 2^{j(2 \beta+(1 / 2))} \varepsilon\right\} \leq \frac{\operatorname{var}\left(\sum_{p=1}^{n} \sum_{i=1}^{r} H_{i, i, n}^{*}\left(Y_{p}, Y_{p}\right)\right)}{n^{2} 2^{j(4 \beta-3)} \varepsilon^{2}} \leqslant \frac{n 2^{j(4 \beta-3)}}{n^{2} 2^{j(4 \beta-3)} \varepsilon^{2}}=n^{-1} 2^{j} \mathcal{\varepsilon}^{-2}, \\
P\left\{\left|J_{n}^{(4)}\right| \geq \frac{2^{j(2 \beta+(1 / 2))} \varepsilon}{n}\right\} \leq \frac{\operatorname{var}\left(\sum_{1 i_{1}<i_{2} \leq r} \sum_{p=1}^{n} H_{i_{1}, i_{2}, n}^{*}\left(Y_{p}, Y_{p}\right)\right)}{n^{2} 2^{j(4 \beta-3)} \varepsilon^{2}} \leqslant \frac{n 2^{j(4 \beta-2)}}{n^{2} 2^{j(4 \beta-3)} \varepsilon^{2}}=\frac{2^{j}}{n \varepsilon^{2}} .
\end{gathered}
$$


If $n \longrightarrow \infty$, then for arbitrary given $\varepsilon>0$, $n^{-1} 2^{j} \mathcal{E}^{-2} \longrightarrow 0$. Moreover, $n 2^{-j(2 \beta+(1 / 2))} J_{n}^{(2)}<\varepsilon \quad$ and $n 2^{-j(2 \beta+(1 / 2))} J_{n}^{(4)}<\varepsilon$, a.s. So $J_{n}$ can be denoted by

$$
\begin{aligned}
n 2^{-j(2 \beta+(1 / 2))} J_{n}= & n^{-1} 2^{-j(2 \beta-(3 / 2))} \sum_{1 \leq i_{1}<i_{2} \leq r} \sum_{1 \leq p_{1}<p_{2} \leq n} H_{i_{1}, i_{2}, n}^{*}\left(Y_{p_{1}}, Y_{p_{2}}\right) \\
& +n^{-1} 2^{-j(2 \beta-(3 / 2))} \sum_{1 \leq i \leq r} \sum_{1 \leq p_{1}<p_{2} \leq n} H_{i, i, n}^{*}\left(Y_{p_{1}}, Y_{p_{2}}\right)+o(1) \\
= & n^{-1} 2^{-j(2 \beta-(3 / 2))} \sum_{1 \leq i_{1} \leq i_{2} \leq r} \sum_{1 \leq p_{1}<p_{2} \leq n} H_{i_{1}, i_{2}, n}^{*}\left(Y_{p_{1}}, Y_{p_{2}}\right)+o(1) .
\end{aligned}
$$

Let

$$
H_{i_{1}, i_{2}, n}\left(Y_{p_{1}}, Y_{p_{2}}\right):=n^{-1} 2^{-j(2 \beta-(3 / 2))+1} H_{i_{1}, i_{2}, n}^{*}\left(Y_{p_{1}}, Y_{p_{2}}\right) \text {, }
$$

so

$$
n 2^{-j(2 \beta+(1 / 2))} J_{n}=\sum_{1 \leq i_{1} \leq i_{2} \leq r} \sum_{1 \leq p_{1}<p_{2} \leq n} H_{i_{1}, i_{2}, n}\left(Y_{p_{1}}, Y_{p_{2}}\right)+o(1) .
$$

It is easy to check that $H_{i_{1}, i_{2}, n}\left(Y_{p_{1}}, Y_{p_{2}}\right)$ are symmetric functions. It is similar to the work of Theorem $A$ in [2] that $E H_{i_{1}, i_{2}, n}^{4}\left(Y_{p_{1}}, Y_{p_{2}}\right)$ and $E G_{i_{1}, i_{2}, n}^{2}\left(Y_{p_{1}}, Y_{p_{2}}\right)$ satisfy the condition of Lemma 3. According to Lemma 2 and Lemma 3,

$$
s_{i_{1}, i_{2}, n}^{-1} \sum_{1 \leq p_{1}<p_{2} \leq n} H_{i_{1}, i_{2}, n}\left(Y_{p_{1}}, Y_{p_{2}}\right) \stackrel{d}{\longrightarrow} N(0,1),
$$

where $s_{i_{1}, i_{2}, n}^{2}:=(1 / 2) n(n-1) E H_{i_{1}, i_{2}, n}^{2}\left(Y_{1}, Y_{2}\right)$.

Thus,

$$
\sum_{1 \leq p_{1}<p_{2} \leq n} H_{i_{1}, i_{2}, n}\left(Y_{p_{1}}, Y_{p_{2}}\right) \stackrel{d}{\longrightarrow} N\left(0, s_{i_{1}, i_{2}, n}^{2}\right) .
$$

The detail discussion is similar to the proof of Theorem A in [2]. So,

$$
\sum_{1 \leq i_{1} \leqslant i_{2} \leq r} \sum_{1 \leq p_{1}<p_{2} \leq n} H_{i_{1}, i_{2}, n}\left(Y_{p_{1}}, Y_{p_{2}}\right) \stackrel{d}{\longrightarrow} N\left(0, \sum_{1 \leq i_{1} \leqslant i_{2} \leq r} s_{i_{1}, i_{2}, n}^{2}\right) .
$$

Consider the nonlinear multiwavelet estimator

$$
\hat{f}_{n}^{\text {non }}(x):=\sum_{i=1}^{r} \sum_{k \in Z} \widehat{c}_{i j_{0} k} \phi_{i j_{0} k}+\sum_{j=j_{0}}^{j_{1}} \sum_{i=1}^{r} \sum_{k \in Z} \hat{d}_{i j k} \psi_{i j k}=\hat{f}_{n}+Q_{j_{0}, j_{1}} \hat{f}_{n}^{\text {non }} \text {. }
$$

Theorem 3. Under the conditions (C1-C3), with $\beta>1$ and $l>\beta+1$, if $f_{X} \in L^{p}(R),(p>2)$, then the nonlinear estimator $\widehat{f}_{n}^{\text {non }}$ satisfies

$$
\begin{aligned}
& n 2^{-j_{0}((2 \beta+(1 / 2))}\left[\left\|\hat{f}_{n}^{\text {non }}-f_{X}\right\|_{2}^{2}-E\left\|\hat{f}_{n}^{\text {non }}-f_{X}\right\|_{2}^{2}\right] \stackrel{d}{\longrightarrow} N\left(0, \sum_{1 \leq i_{1} \leq i_{2} \leq r} s_{i_{1}, i_{2}, n}^{2}\right), \\
& \longrightarrow \infty, \text { and } n^{-1} 2^{j_{1}(4 \beta+4)} \longrightarrow 0, \\
& \longrightarrow f_{Y}(y)=\int_{y-1}^{y} \frac{1}{\sqrt{2 \pi}} e^{-\left(x^{2} / 2\right)} \mathrm{d} x, \quad-\infty<y<\infty .
\end{aligned}
$$

with $\lambda_{j} \sim(j / n) 2^{j \beta}, j_{0}, i_{1} \longrightarrow \infty$, and $n^{-1} 2^{j_{1}(4 \beta+4)} \longrightarrow 0$, where $s_{i_{1}, i_{2}, n}^{2}$ : $=(1 / 2) n(n-1) E H_{i_{1}, i_{2}, n}^{2}\left(Y_{1}, Y_{2}\right)$ and $H_{i_{1}, i_{2}, n}^{2}\left(Y_{p_{1}}, Y_{p_{2}}\right)$ are defined by equations (55) and (62).

The proof is similar to Theorem $B$ in [2].

\section{Numerical Example}

In this section, an example is given for discussing the results of multiwavelet deconvolution density estimators.

Choose the model $Y=X+\varepsilon$. Construct the data $X$ by the function "randn" and error data $\varepsilon$ by the function "rand" in Matlab. That is, $X \sim N(0,1)$ is a standard normal random variable and $\varepsilon \sim U(0,1)$ is a uniform random variable. So $f_{Y}$ is the convolution of $f_{X}$ and $f_{\varepsilon}$, where $f_{X}(x)=(1 / \sqrt{2 \pi}) e^{-\left(x^{2} / 2\right)}$ and $f_{\varepsilon}(z)=\left\{\begin{array}{ll}1 & 0<z<1 \\ 0 & \text { else }\end{array}\right.$.

By the formula of the convolution, we have density function $f_{Y}$ of random variable $Y$ as follows:
In Figure 1, random data $Y$ is shown at the left side and its sampling number is 2048. At the right side of Figure 1, the blue dotted curve denotes the empirical density of data $Y$ and the density of data $Y$ is shown by the red solid curve.

According to Theorem 1 and $E \widehat{f}_{n}(x)=P_{j} f_{X}$, we choose the multiwavelet $\mathrm{Sa} 4$ to estimate the expectation of the linear multiwavelet deconvolution density estimators. The sampling data are decomposed into 4 levels by multiwavelet transform.

The density $f_{X}$ of $X$ is shown in the second row and second column of Figure 2. In the first row and first column of Figure 2, the linear multiwavelet deconvolution density estimator $\widehat{f}_{n}$ of $X$ is given by the black solid line and the expectation $E \hat{f}_{n}$ of linear multiwavelet deconvolution density estimator $\hat{f}$ defined by equation (12) is shown by the red solid line. In the first row and second column of Figure 2, 


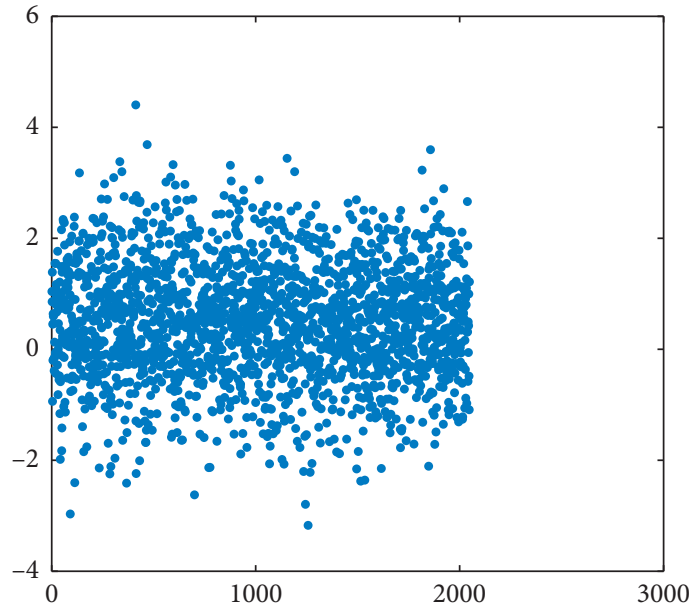

(a)

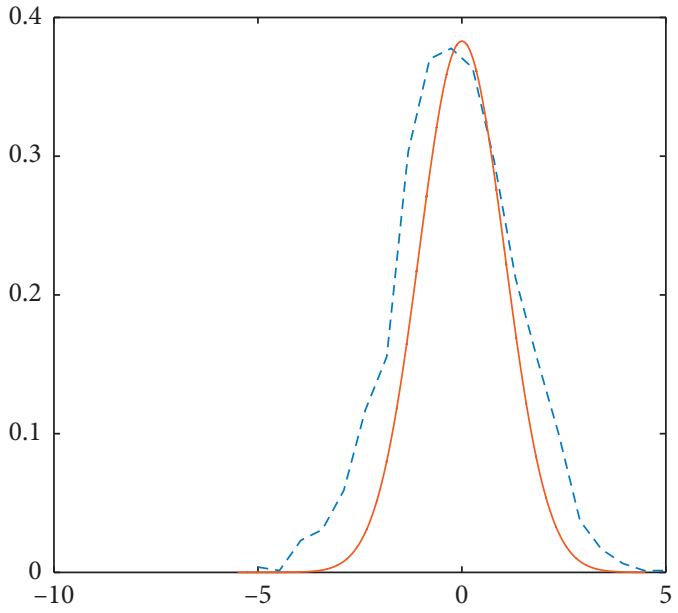

(b)

Figure 1: (a) Data Y; (b) empirical density and density of $Y$.

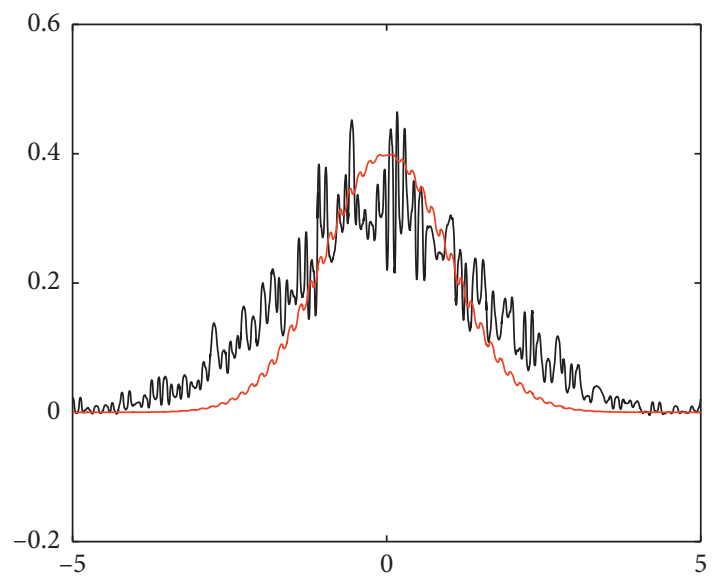

(a)

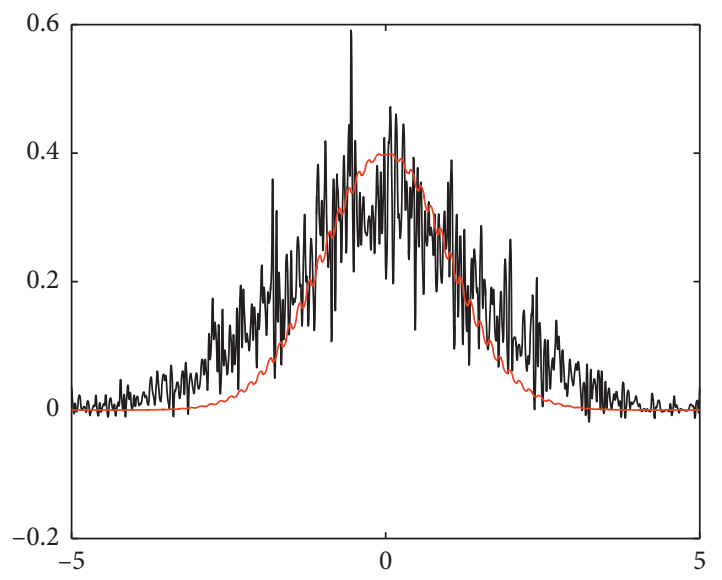

(c)

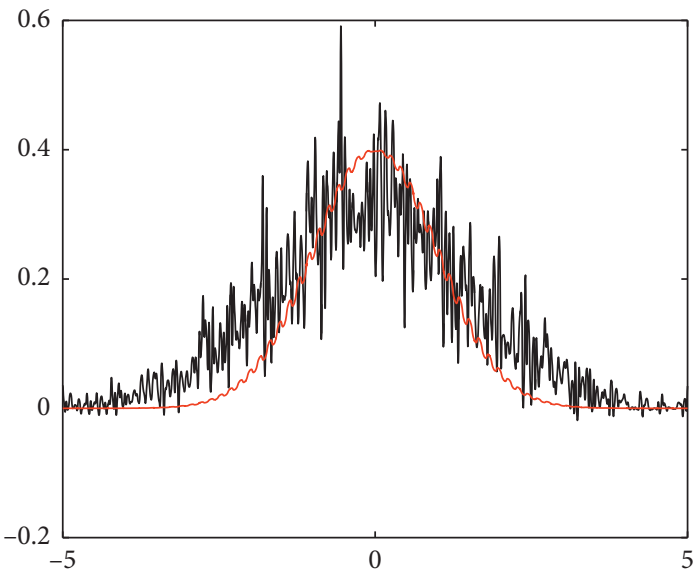

(b)

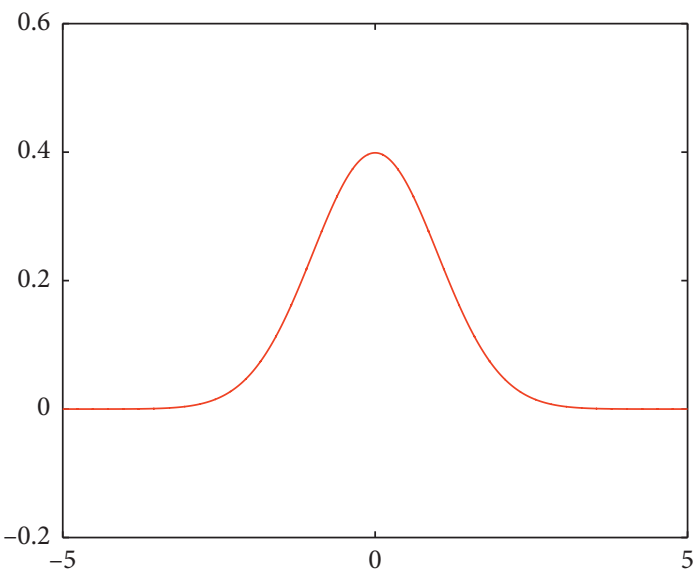

(d)

Figure 2: Multiwavelet deconvolution density estimators.

nonlinear multiwavelet deconvolution density estimator $\widehat{f}_{1 n}^{\text {non }}$ is given by the black solid line and the expectation $E \widehat{f}_{1 n}^{\text {non }}$ of nonlinear multiwavelet density estimator $\widehat{f}_{1 n}^{\text {non }}$ is shown by the red solid line, where $\hat{f}_{1 n}^{\text {non }}$ can be denoted by

$$
\widehat{f}_{1 n}^{\text {non }}(x):=\sum_{i=1}^{r} \sum_{k \in Z} \widehat{c}_{i j_{0} k} \phi_{i j_{0} k}+\sum_{i=1}^{r} \sum_{k \in Z} \widehat{d}_{i j_{0} k} \psi_{i j_{0} k} \text {. }
$$


TABLE 1: Asymptotic normality of multiwavelet density estimators.

\begin{tabular}{lcc}
\hline Estimators & $P$ value & Results of normality \\
\hline$\hat{f}_{n}-f_{X}$ & 0.5000 & 0 \\
$\hat{f}_{1 n}^{\text {non }}-f_{X}$ & 0.4642 & 0 \\
$\widehat{f}_{2 n}^{\text {non }}-f_{X}$ & 0.5000 & 0 \\
\hline
\end{tabular}

Note: this table shows the results of the J-B test for multiwavelet estimators $\widehat{f}_{n}-f_{X}, \widehat{f}_{1 n}^{\text {non }}-f_{X}$, and $\widehat{f}_{2 n}^{\text {non }}-f_{X}$. If the result of Jarque-Bera test is zero, it indicates that it obeys normal distribution at significant level 0.05 . If $P$ value of the Jarque-Bera test is closer to zero, it indicates that the original assumption of normal distribution can be rejected.

In the second row and first column of Figure 2 , nonlinear multiwavelet deconvolution density estimator $\widehat{f} \widehat{f}_{2 n}^{\text {non }}$ is given by the black solid line and the expectation $E \widehat{f}_{2 n}^{\text {non }}$ of nonlinear multiwavelet density estimator $\widehat{f}_{2 n}^{\text {ton }}$ is shown by the red solid line, where $\widehat{f}_{2 n}^{\text {non }}$ can be denoted by

$$
\hat{f}_{2 n}^{\text {non }}(x):=\sum_{i=1}^{r} \sum_{k \in Z} \widehat{c}_{i j_{0} k} \phi_{i j_{0} k}+\sum_{j=j_{0}}^{j_{0}+1} \sum_{i=1}^{r} \sum_{k \in Z} \hat{d}_{i j k} \psi_{i j k} .
$$

Moreover, asymptotic normality is identified by the Jarque-Bera test. The results of the J-B test are given for $\widehat{f}_{n}-f_{X}, \widehat{f}_{1 n}^{\text {non }}-f_{X}$, and $\widehat{f}_{2 n}^{\text {non }}-f_{X}$ in Table 1 .

In Table 1, all results of normality are zero, and the original assumption of normal distribution can be accepted by the $P$ value of the Jarque-Bera test. These imply the conclusions of Theorem 2 and Theorem 3.

\section{Data Availability}

No data were used to support this study.

\section{Conflicts of Interest}

The authors declare that they have no conflicts of interest.

\section{Acknowledgments}

This work was supported by the Planning of Philosophy and Social Sciences in Zhejiang Province of China (19NDQN340YB) and National Natural Science Foundation of China (11771100).

\section{References}

[1] X. Zeng, "A note on wavelet deconvolution density estimation," International Journal of Wavelets, Multiresolution and Information Processing, vol. 15, no. 6, Article ID 1750055, 2017.

[2] Y. Liu and X. Zeng, "Asymptotic normality for wavelet deconvolution density estimators," Applied and Computational Harmonic Analysis, vol. 48, no. 1, pp. 321-342, 2020.

[3] H. Guo and Y. Liu, "Wavelet estimations for densities and their derivatives with Fourier oscillating noises," Journal of Inequalities and Applications, vol. 2014, no. 1, p. 236, 2014.

[4] E. Giné and W. R. Madych, "On wavelet projection kernels and the integrated squared error in density estimation," Statistics \& Probability Letters, vol. 91, pp. 32-40, 2014.

[5] R. Li and Y. Liu, "Wavelet optimal estimations for a density with some additive noises," Applied and Computational Harmonic Analysis, vol. 36, no. 3, pp. 416-433, 2014.
[6] L. Shen, H. H. Tan, and J. Y. Tham, "Some properties of symmetric-antisymmetric orthonormal multiwavelets," IEEE Transactions on Signal Processing, vol. 48, no. 7, pp. 2161-2163, 2000.

[7] J. Lian and C. K. Chui, "Balanced multiwavelets with short filters," IEEE Signal Processing Letters, vol. 11, no. 2, pp. 75-78, 2004.

[8] C. K. Chui, An Introduction to Wavelets, Academic, Boston, MA, USA, 1992.

[9] I. Daubechies, Ten Lectures on Wavelets, SIAM, Philadelphia, PA, USA, 1992. 